\title{
Stability and discretisation in delayed and anticipatory systems of applied mechanics
}

\author{
P. B. Béda ${ }^{1}$
}

(Received 9 January 2012; revised 8 June 2012)

\begin{abstract}
In applied mechanics several articles concentrated on the comparison of delayed and non-delayed approaches of controlled machines. We study both continuous and discrete time systems using both numeric and analytic methods. The principal points of interest in the following work are how continuous time systems differ from a representation in some discrete time system in both stability and robustness, and how the discretisation of a continuous time subsystem influences the stability properties of the coupled system.
\end{abstract}

\section{Contents}

\section{Introduction}

C341

http://journal.austms.org.au/ojs/index.php/ANZIAMJ/article/view/5095 gives this article, (c) Austral. Mathematical Soc. 2012. Published July 12, 2012. ISSN 1446-8735. (Print two pages per sheet of paper.) Copies of this article must not be made otherwise available on the internet; instead link directly to this URL for this article. 
2 Discrete time systems

3 Stability of discretised systems

C345

4 Summary

C348

References

C352

\section{Introduction}

The stability of controlled mechanical systems is important. In numerous problems of mechanical engineering a machine is controlled by a digital device to perform some task. Such a system has two essentially different parts. One is the machine, in the sense of mechanical engineering. It is usually described as a continuous time system by using one of the traditional methods of applied mechanics. The other subsystem is the discrete controller. Generally we have a complex nonlinear system consisting of a continuous time and a discrete time subsystems. Instabilities may arise from either the continuous or the time discrete parts. For example, in balancing, the unstable continuous time system should be stabilised by the digital control. An obvious problem in such systems is the sampling delay effect. When it is neglected an anticipatory model is obtained. The properties of this model may or may not be different from the original one.

Previously [1] I derived the equation of motion for a simple controlled inverted pendulum with length $l$ and mass $m$ (see Figure 1). The pendulum was attached to a cart of mass $M$ with a hinge and its stability was achieved by applying a force $F$ to the cart. Such a balancing problem is widely studied $[2,6,7,8,9]$ because it is a simple way to study how to keep a system in the vicinity of an unstable equilibrium position. 


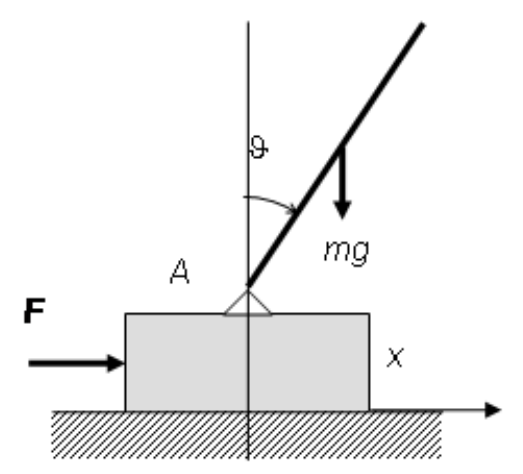

Figure 1: Inverted pendulum.

By using a Lagrangian formalism the equation of motion is

$$
\begin{aligned}
& \frac{1}{3} m l^{2} \ddot{\vartheta}+\frac{1}{2} m l \ddot{x} \cos \vartheta-\frac{1}{2} m g l \sin \vartheta=0, \\
& m \ddot{x}+\frac{1}{2} m l \ddot{\vartheta} \cos \vartheta-\frac{1}{2} m l^{2} \sin \vartheta=Q(t),
\end{aligned}
$$

where the two generalised coordinates are the position $x$ of the cart and the angular position $\vartheta$ of the pendulum measured from the upwards vertical. On the right hand side of (2) the generalised force $\mathrm{Q}$ is equal to the control force

$$
\mathrm{Q}(\mathrm{t}) \equiv \mathrm{F}(\mathrm{t})=\mathrm{c}_{1} \dot{\vartheta}(\mathrm{t}-\tau)+\mathrm{c}_{0} \vartheta(\mathrm{t}-\tau)
$$

In (3) the output of the controller is delayed expressing that in most cases there is a time delay $\tau$ between the measurement or sampling and the action of the controller.

Beda [1] studied the stability of the continuous time system with numerical analysis. Now, analytic methods are used. Our aim is to get the exact location of stability domains of the discrete time systems in the form of a stability chart in the plane of the forcing parameters. 


\section{Discrete time systems}

Two possible methods were described in previous work [1]. Firstly, we may express $\ddot{x}$ from (2) and substitute into (1) so that

$$
\begin{aligned}
& \ddot{\vartheta}=-\frac{3 \sin \vartheta \cos \vartheta}{4-3 \cos ^{2} \vartheta} \dot{\vartheta}^{2}+\frac{6 g \sin \vartheta}{\left(4-3 \cos ^{2} \vartheta\right) l}-\frac{\cos \vartheta}{\left(4-3 \cos ^{2} \vartheta\right) m l} F(t), \\
& F(t)=c_{1} \dot{\vartheta}(t-\tau)+c_{0} \vartheta(t-\tau) .
\end{aligned}
$$

The other possibility is to keep the two generalised coordinates and force F, an unknown function of the form (3), and then

$$
\begin{aligned}
\ddot{\vartheta} & =\frac{3 l \dot{x}^{2} \sin \vartheta \cos \vartheta-6 g \sin \vartheta}{\left(-4+3 \cos ^{2} \vartheta\right) l}+\frac{6 \mathrm{~F} \cos \vartheta}{\left(-4+3 \cos ^{2} \vartheta\right) \mathrm{ml}}, \\
\ddot{x} & =\frac{3 g \sin \vartheta \cos \vartheta-2 l \dot{x}^{2} \sin \vartheta}{\left(-4+3 \cos ^{2} \vartheta\right)}-\frac{4 \mathrm{~F}}{\left(-4+3 \cos ^{2} \vartheta\right) \mathrm{m}}, \\
\mathrm{F}(\mathrm{t}) & =c_{1} \dot{\vartheta}(t-\tau)+c_{0} \vartheta(t-\tau) .
\end{aligned}
$$

A detailed derivation of the continuous time dynamical systems (4), and (6) was performed by Beda [1] and it was followed by a linear stability investigation of the upright position. Then the behaviour of the systems with delayed and non-delayed control is compared using numerical analysis, which requires discretisation.

When delay is omitted in the control law $(5)(\tau=0)$ an incursive feed-in-time system is obtained [3]:

$$
\mathrm{F}(\mathrm{t})=\mathrm{c}_{1} \dot{\vartheta}(\mathrm{t})+\mathrm{c}_{0} \vartheta(\mathrm{t}),
$$

which is more obvious for a discrete time $t \in\left\{t_{0}, t_{1}, \ldots, t_{i}, \ldots\right\}$ system

$$
F\left(t_{i}\right)=c_{1} \dot{\vartheta}\left(t_{i}\right)+c_{0} \vartheta\left(t_{i}\right),
$$


while any nonzero delay should be interpreted as some recursive form. Assume for the sake of simplicity that

$$
\mathrm{t}_{\mathrm{i}}=\mathrm{t}_{0}+\mathrm{i} \Delta \mathrm{t}, \quad \text { where } \quad \mathrm{i}=1,2, \ldots,
$$

and $\tau=\Delta t$, where $\Delta t$ is a small positive time step. Then (5) produces the recursion

$$
F\left(t_{i}\right)=c_{1} \dot{\vartheta}\left(t_{i-1}\right)+c_{0} \vartheta\left(t_{i-1}\right) .
$$

When numerical simulation is needed, we should form a set of difference equations instead of (5), (4) and (6). Let us introduce new variables:

$$
y_{1}=\vartheta, \quad y_{2}=\dot{\vartheta}, \quad y_{3}=x, \quad y_{4}=\dot{x}, \quad y_{5}=F,
$$

and simplify the notation,

$$
y_{k}(i)=y_{k}\left(t_{i}\right), \quad k=1,2, \ldots, 5 .
$$

Then in the feed-in-time $(\tau=0)$ case from (4) and (7)

$$
\begin{aligned}
y_{1}(i+1)= & y_{1}(i)+\Delta t y_{2}(i) \\
y_{2}(i+1)= & y_{2}(i)+\left(-\frac{3 \sin y_{1}(i) \cos y_{1}(i)}{4-3 \cos ^{2} y_{1}(i)}\left(y_{2}(i)\right)^{2}+\frac{6 g \sin y_{1}(i)}{\left(4-3 \cos ^{2} y_{1}(i)\right) l}\right. \\
& \left.-\frac{6 \cos y_{1}(i)}{\left(4-3 \cos ^{2} y_{1}(i)\right) m l} y_{5}(i)\right) \Delta t \\
y_{5}(i+1)= & c_{1} y_{2}(i+1)+c_{0} y_{1}(i+1),
\end{aligned}
$$

is obtained. Or, more simply,

$$
\begin{aligned}
y_{1}(i+1)= & y_{1}(i)+\Delta t y_{2}(i) \\
y_{2}(i+1)= & y_{2}(i)+\left(-\frac{3 \sin y_{1}(i) \cos y_{1}(i)}{4-3 \cos ^{2} y_{1}(i)}\left(y_{2}(i)\right)^{2}+\frac{6 g \sin y_{1}(i)}{\left(4-3 \cos ^{2} y_{1}(i)\right) l}\right. \\
& \left.-\frac{6 c_{1} \cos y_{1}(i)}{\left(4-3 \cos ^{2} y_{1}(i)\right) m l} y_{2}(i)-\frac{6 c_{0} \cos y_{1}(i)}{\left(4-3 \cos ^{2} y_{1}(i)\right) m l} y_{1}(i)\right) \Delta t
\end{aligned}
$$


From (6) the recursive discrete dynamical system reads

$$
\begin{aligned}
y_{1}(i+1)= & y_{1}(i)+\Delta t y_{2}(i), \\
y_{2}(i+1)= & y_{2}(i)+\left(\frac{3 y_{4}^{2}(i) l \sin y_{1}(i) \cos y_{1}(i)-6 g \sin y_{1}(i)}{\left(-4+3 \cos ^{2} y_{1}(i)\right) l}\right. \\
& \left.+\frac{6 \cos y_{1}(i) y_{5}(i)}{\left(-4+3 \cos ^{2} y_{1}(i)\right) m l}\right) \Delta t, \\
y_{3}(i+1)= & y_{3}(i)+\Delta t y_{4}(i), \\
y_{4}(i+1)= & y_{4}(i)+\left(\frac{3 g \sin y_{1}(i) \cos y_{1}(i)-2 y_{4}^{2}(i) l \sin y_{1}(i)}{\left(-4+3 \cos ^{2} y_{1}(i)\right)}\right. \\
& \left.-\frac{4 y_{5}(i)}{\left(-4+3 \cos ^{2} y_{1}(i)\right) m}\right) \Delta t, \\
y_{5}(i+1)= & c_{1} y_{2}(i)+c_{0} y_{1}(i) .
\end{aligned}
$$

Previously [1] the stability of the equilibrum position $\theta=0$ was investigated using linear approximations of (4) and (5). Then the discrete time systems were studied using numerical simulations. Now the mapping obtained by discretisation will be studied.

\section{$3 \quad$ Stability of discretised systems}

The linear stability of a mapping can generally be investigated by studying the eigenvalues $\lambda_{i}$ of its monodromy operator $[4,5]$. For the anticipatory case, from the linearisation of the right-hand-side of (9),

$$
\begin{aligned}
& y_{1}(i+1)=y_{1}(i)+\Delta t y_{2}(i), \\
& y_{2}(i+1)=y_{2}(i)+\left(\frac{6 g y_{1}(i)}{l}-\frac{6 c_{1}}{m l} y_{2}(i)-\frac{6 c_{0}}{m l} y_{1}(i)\right) \Delta t
\end{aligned}
$$

The monodromy operator reads

$$
A=\left[\begin{array}{cc}
1 & \Delta t \\
6 \Delta t g / l-6 c_{0} \Delta t / m l & 1-6 c_{1} \Delta t / m l
\end{array}\right] .
$$




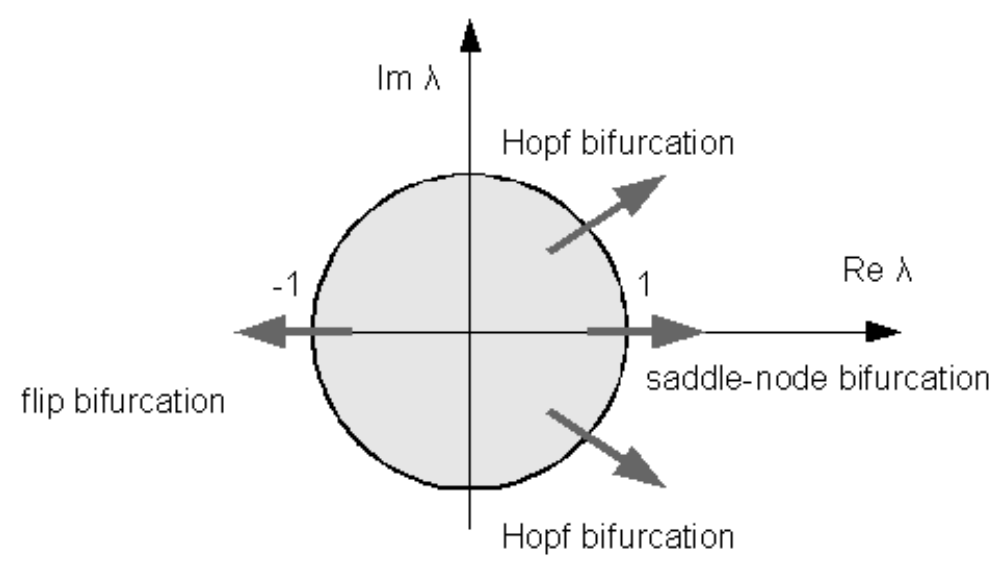

Figure 2: Stability boundary in the plane of complex eigenvalues.

Its characteristic equation is

$$
\lambda^{2}+2 \frac{\left(-m l+3 c_{1} \Delta t\right)}{m l} \lambda-\frac{6(\Delta t)^{2} g m-6(\Delta t)^{2} c_{0}-m l+6 c_{1}(\Delta t)}{m l}=0 .
$$

The solutions are eigenvalues

$$
\lambda_{1,2}=1-3 \widetilde{\mathbf{c}}_{1} \Delta t \pm \sqrt{\left(3 \widetilde{\mathbf{c}}_{1} \Delta t\right)^{2}+6(\Delta t)^{2}\left(\alpha^{2}-\widetilde{\mathbf{c}}_{0}\right)}
$$

where

$$
\widetilde{\mathbf{c}}_{1}=\frac{\mathrm{c}_{1}}{\mathrm{ml}}, \quad \widetilde{\mathrm{c}}_{0}=\frac{\mathrm{c}_{0}}{\mathrm{ml}} \quad \text { and } \quad \alpha^{2}=\frac{\mathrm{g}}{\mathrm{l}} .
$$

The stability condition is $|\lambda|<1$ for all solutions of (11).

In the complex plane of eigenvalues (see Figure 2) the stable region is in the unit circle and loss of stability happens when one of the eigenvalues leaves the unit circle. The regions of three possible types of instabilities, the flip, saddle-node and Hopf bifurcations, are also shown in Figure 2. For $\Delta t \neq 0$ this result is obviously the same as that derived by Beda [1] for the continuous time case. 
In discrete time systems with time step delay $\tau=\Delta t$ the stability analysis is much simpler than using the theorem cited by Beda [1]. For such a recursive system obtained from (10) the monodromy operator is

$$
\mathbf{B}=\left[\begin{array}{ccccc}
1 & \Delta \mathrm{t} & 0 & 0 & 0 \\
6 \Delta \mathrm{tg} / \mathrm{l} & 1 & 0 & 0 & -6 \Delta \mathrm{t} / \mathrm{ml} \\
0 & 0 & 1 & \Delta \mathrm{t} & 0 \\
-3 \Delta \mathrm{tg} / \mathrm{l} & 0 & 0 & 1 & 4 \Delta \mathrm{t} / \mathrm{m} \\
c_{0} & c_{1} & 0 & 0 & 0
\end{array}\right]
$$

Its characteristic equation is

$$
\operatorname{det}(\mathbf{B}-\lambda \mathbf{I})=0,
$$

where $\mathbf{I}$ denotes identity operator as usual. Introducing $\varepsilon=-1+\lambda$, (12) is rewritten as

$$
\operatorname{det}\left[\begin{array}{ccccc}
-\varepsilon & \Delta \mathrm{t} & 0 & 0 & 0 \\
6 \Delta \mathrm{tg} / \mathrm{l} & -\varepsilon & 0 & 0 & -6 \Delta \mathrm{t} / \mathrm{ml} \\
0 & 0 & -\varepsilon & \Delta \mathrm{t} & 0 \\
-3 \Delta \mathrm{tg} / \mathrm{l} & 0 & 0 & -\varepsilon & 4 \Delta \mathrm{t} / \mathrm{m} \\
c_{0} & c_{1} & 0 & 0 & -\varepsilon-1
\end{array}\right]=0
$$

and thus

$$
\varepsilon^{5}+\varepsilon^{4}-6 \frac{\Delta t\left(-c_{1}+\Delta \operatorname{tgm}\right) \varepsilon^{3}}{m l}-6 \frac{(\Delta t)^{2}\left(-c_{0}+g m\right) \varepsilon^{2}}{m l}=0 .
$$

Here, $\varepsilon=0$, that is, $\lambda=1$, is solution of multiplicity two, thus

$$
\begin{array}{r}
(-1+\lambda)^{3}+(-1+\lambda)^{2}-6 \frac{\Delta \mathrm{t}\left(-\mathrm{c}_{1}+\Delta \mathrm{tgm}\right)(-1+\lambda)}{\mathrm{ml}} \\
-6 \frac{(\Delta \mathrm{t})^{2}\left(-\mathrm{c}_{0}+\mathrm{gm}\right)}{\mathrm{ml}}=0
\end{array}
$$

should be solved for $\lambda$ to find the remaining roots. 
By fixing $\Delta t=0.005$ and $\alpha=1$ we have

$$
(-1+\lambda)^{3}+(-1+\lambda)^{2}-6\left(0.005-\widetilde{\mathfrak{c}}_{1}\right)(-1+\lambda)+0.000150 \widetilde{\mathfrak{c}}_{0}-0.000150=0 .
$$

The solutions are plotted in Figures 3 and 4 as functions of $\operatorname{ch} 0=\mathrm{c}_{0} / \mathrm{ml}$ and $\operatorname{ch} 1=\mathrm{c}_{1} / \mathrm{ml}$.

By comparing Figures 3 and 4 we find that the results for eigenvalue $\lambda_{1}$ are visibly similar: in both cases they are larger then one at the origin and near to the axis $\widetilde{\mathbf{c}}=0$. We also observe that in the anticipatory case, $\lambda_{1}$ decreases "faster" for $\widetilde{\mathbf{c}}_{1}>0$. The main difference is in the form of the surfaces calculated for the other eigenvalues, because in the recursive case we see a stability limit for $\widetilde{\mathbf{c}}_{1}>0$.

To find the exact stability boundaries for (11) and (13) we substitute

$$
\lambda=\beta \pm \sqrt{1-\beta^{2}}, \quad 0 \leqslant \beta \leqslant 1,
$$

into (11) and (13) and solve for $\widetilde{\mathbf{c}}_{1}$ and $\widetilde{\mathbf{c}}_{0}$. Then the stability charts presented in Figure 5 are obtained.

Here the main difference is that for the anticipatory system there is no upper boundary for the stable region in both directions, while in the case of the recursive system the stable region is bounded. In this sense stability properties are weaker in the recursive case.

\section{Summary}

When a mechanical system is controlled by some digital device the finite time step causes a decrease in the stable region of the control parameter plane. For this reason we should be careful in modelling. In correct modelling we should keep the control force separate in order to see the real nature of control. From Equations (9) and (8) we see how incursion is hidden, when delay is omitted. Then a hidden anticipatory effect may result in better stability properties and 

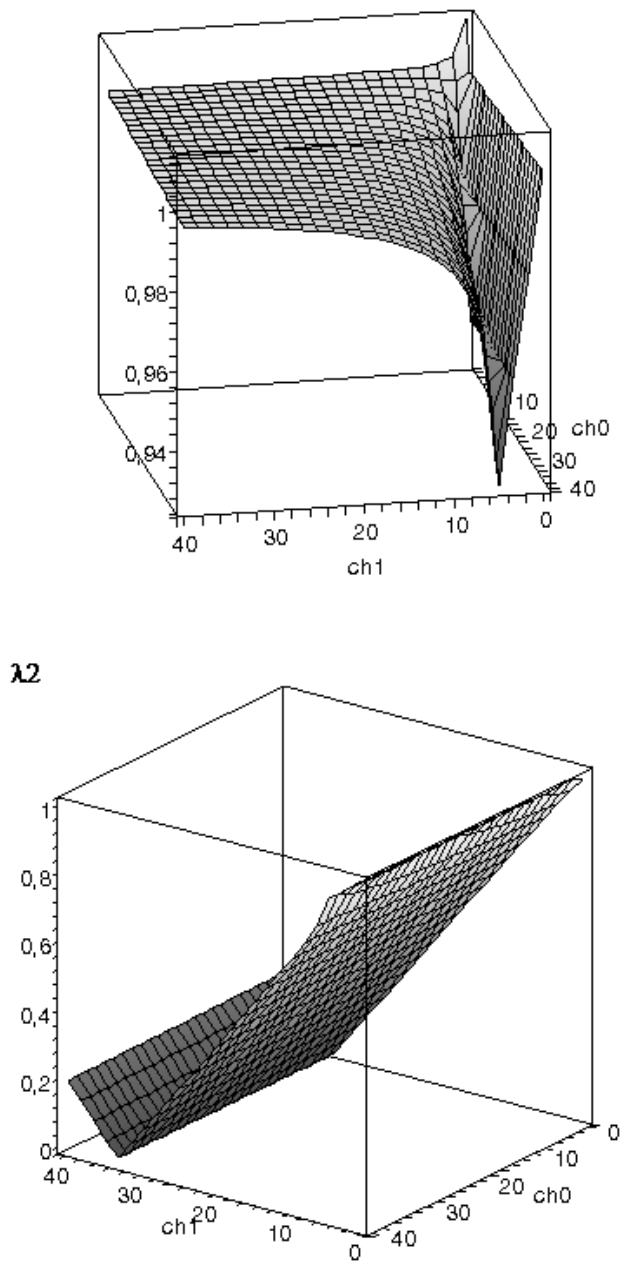

Figure 3: The eigenvalues at anticipatory case $\lambda_{i}=\lambda_{i}(\operatorname{ch} 0, \operatorname{ch} 1)$. 

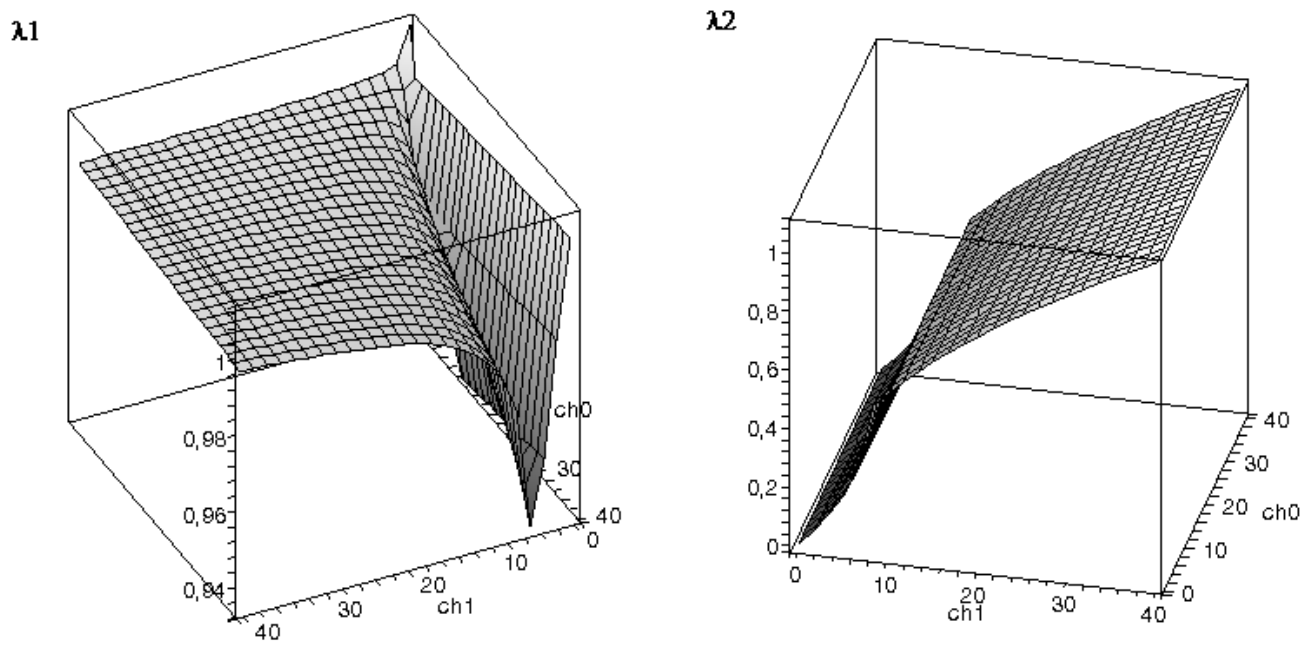

\section{$\lambda 3$}

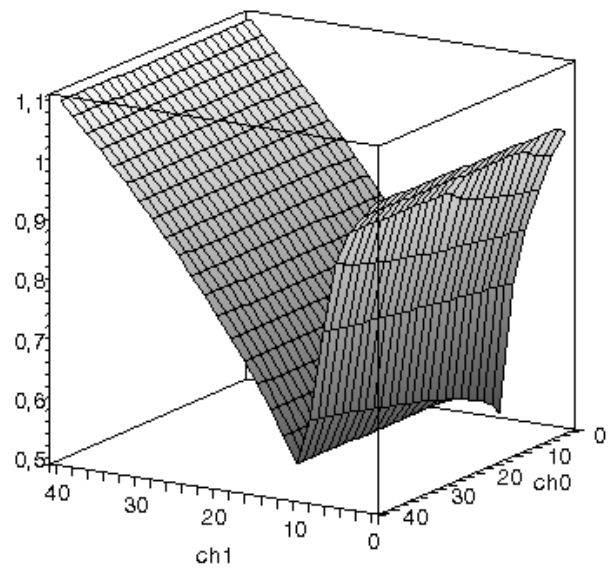

Figure 4: The eigenvalues at recursive case $\lambda_{i}=\lambda_{i}(\operatorname{ch} 0, \operatorname{ch} 1)$. 


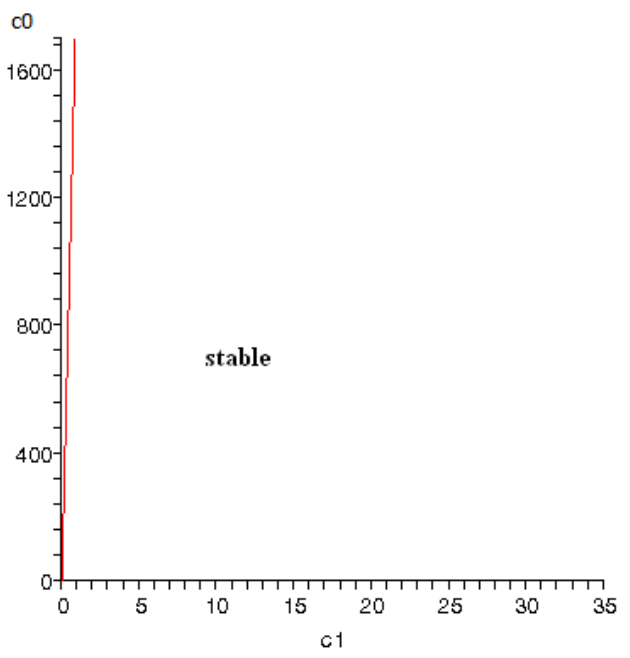

anticipatory

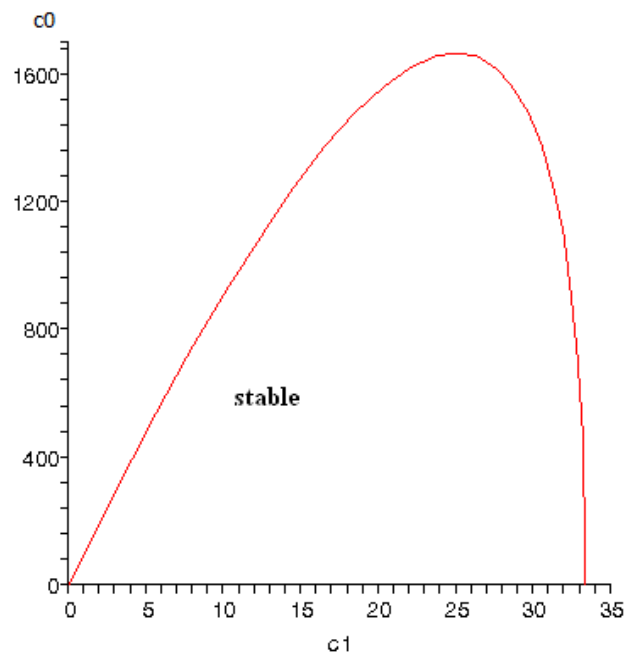

recursive

Figure 5: Stability charts in $\left(\widetilde{\mathfrak{c}}_{1}, \widetilde{\mathfrak{c}}_{0}\right)$ plane.

a larger stability region for the model. This may lead to unexpected unstable behaviour of the real system.

As we have seen, stability investigation leads to the same results in both continuous and discrete time systems for anticipatory models. The reason is that at feed-in-time the behaviour of the system can be described by a set of first order ordinary differential equations. When sampling delay is taken into consideration we should use functional differential equations, which introduces many complications. In our case a recursive discrete time system was formed and the stability region was found by a simple analytic method.

The recursive system has a double critical eigenvalue. It shows that the system is on a stability boundary, but the physical system is a free system in coordinate $x$ and this is the source of such an instability. 
Acknowledgements This work was supported by the National Scientific Research Funds of Hungary (OTKA contract number K81531)

\section{References}

[1] P. B. Béda. On delayed and anticipatory systems in applied mechanics. In D.M. Dubois, editor, Computing Anticipatory Systems: CASYS 2009 International Conference, volume 1303 of AIP Conference Proceedings, pages 363-370. American Institute of Physics, New York, 2010. doi:10.1063/1.3527174 C341, C342, C343, C345, C346, C347

[2] G. Stépán. Retarded Dynamical Systems: Stability and Characteristic Functions. Longman, Harlow, 1989. C341

[3] D. M. Dubois. Incursive anticipatory control of a chaotic robot arm. In D.M. Dubois, editor, Computing Anticipatory Systems: CASYS 97 International Conference, volume 437 of AIP Conference Proceedings, pages 406-417. American Institute of Physics, New York, 1998. doi:10.1063/1.56337 C343

[4] Insperger, T., Stépán, G. and Turi, J., Delayed feedback of sampled higher derivatives, Phil. Trans. Royal Soc. A, 368, 2010, 469-482. doi:10.1098/rsta.2009.0246 C345

[5] Troger, H. and Steindl, A. Nonlinear stability and bifurcation theory, An introduction for scientists and engineers. Springer-Verlag, Wien, New York 1990. C345

[6] Atay, F. Balancing the inverted pendulum using position feedback, Appl. Math. Lett., 12, 1999, 51-56. doi:10.1016/S0893-9659(99)00056-7 C341

[7] Cabrera, J. and Milton, J. Human stick balancing: Tuning Levy flights to improve balance control, Chaos, 14, 2004, 691-698.

doi:10.1063/1.1785453 C341 
[8] Stépán, G. and Kollár,L. Balancing with reflex delay, Math. Comput. Modelling, 31, 2000, 199-205. doi:10.1016/S0895-7177(00)00039-X C341

[9] Sieber J. and Krauskopf, B. Bifurcation analysis of an inverted pendulum with delayed feedback control near a triple-zero eigenvalue singularity, Nonlinearity, 17, 2004, 85-103.

doi:10.1088/0951-7715/17/1/006 C341

\section{Author address}

1. P. B. Béda, HAS-BUTE Research Group on Dynamics of Machines and Vehicles, Budapest University of Technology, Bertalan L. 2. H-1111 Budapest, Hungary.

mailto : bedap@kme. bme.hu 